\title{
Dinâmica Populacional de Glycaspis brimblecombei e Inimigos Naturais em Eucalyptus spp., Cuiabá-MT
}

\author{
Alex Lima da Silva ${ }^{1}$, Otávio Peres-Filho ${ }^{1}$, Alberto Dorval' ${ }^{1}$, \\ Cibele Kotsubo da Cunha e Castro ${ }^{1}$
}

${ }^{1}$ Faculdade de Engenharia Florestal - FENF, Universidade Federal de Mato Grosso - UFMT, Cuiabá/MT, Brasil

\begin{abstract}
RESUMO
Neste trabalho, estudou-se a dinâmica populacional de Glycaspis brimblecombei e seus inimigos naturais em diversos hospedeiros de Eucalyptus spp., na Fazenda Jardim, no município de Cuiabá-MT, no período de um ano. As coletas foram realizadas com armadilhas adesivas de coloração amarela nas folhas e nos ramos das plantas, em cinco talhões. Determinou-se a flutuação populacional da praga e dos seus inimigos naturais, correlacionando-se com variáveis meteorológicas. A flutuação populacional de G. brimblecombei apresentou maior ocorrência de indivíduos no período da seca, com correlação diretamente proporcional à temperatura máxima e inversamente proporcional à umidade relativa do ar, com maiores quantidades de indivíduos nos meses de agosto e setembro de 2008. Psyllaephagus bliteus e outros inimigos naturais não apresentaram correlação com as variáveis meteorológicas. A espécie E. camaldulensis e o clone MG1277 favoreceram o aumento da população de G. brimblecombei, sendo o Clone GG100 o hospedeiro menos favorável ao aumento da população.
\end{abstract}

Palavras-chave: controle biológico, sinecologia, psilídeo-de-concha.

\section{Population Dynamics of Glycaspis brimblecombei and Natural Enemies in Eucalyptus spp. in Cuiaba, State of Mato Grosso, Brazil}

\begin{abstract}
This study presents a one-year investigation on the population dynamics of Glycaspis brimblecombei and their natural enemies in several hosts of Eucalyptus spp. in a farm in the municipality of Cuiaba, State of Mato Grosso, Brazil. Collection was performed by yellow sticky traps and on the leaves and stems of plants in five plots. The population fluctuation of the pest and its natural enemies were determined in correlation with meteorological parameters. The population fluctuation of Glycaspis brimblecombei presented greater occurrence in the dry season, in direct correlation with the maximum temperature and inversely proportional to the relative humidity, showing greater amount of individuals in August and September 2008. Psyllaephagus bliteus and other natural enemies founded in the 'Jardim' farm were not correlated with meteorological parameters. The species E. camaldulensis and the Clone MG1277 favored the population increase of G. brimblecombei, while the Clone GG100 was the least favorable host for the population increase of the pest.
\end{abstract}

Keywords: biological control, synecology, red gum lerp psyllid. 


\section{INTRODUÇÃO}

O psilídeo-de-concha, Glycaspis brimblecombei, é uma praga de origem australiana e de hábito sugador, caracterizado por alimentar-se somente de eucalipto; é de fácil reconhecimento no campo em razão de sua secreção açucarada, em forma de concha, localizada nas folhas das plantas.

No Brasil, esta praga foi detectada no ano de 2003 no Estado de São Paulo, trazendo danos, como: secamento dos ponteiros, redução da atividade fotossintética da planta e redução no crescimento das árvores, além de favorecer a ocorrência de pragas secundárias e o desenvolvimento de um fungo saprófito fumagina (Wilcken et al., 2003).

As espécies E. camaldulensis e E. tererticonis, e o híbrido Urograndis oferecem condições favoráveis ao desenvolvimento e à reprodução de G. brimblecombei, porém E. camaldulensis é o hospedeiro preferido, atualmente (Firmino, 2004).

Tendo-se em vista que atualmente essa praga encontra-se disseminada em todo o Estado de Mato Grosso e que se mostra importante o estudo das populações do psilídeo-de-concha em florestas de Eucalyptus spp., objetivou-se estudar a dinâmica populacional de Glycaspis brimblecombei e de seus inimigos naturais em povoamentos florestais de Eucalyptus camaldulensis, nos clones 'GG100' (Eucalyptusurophylla $\times$ Eucalyptusgrandis)e'MG1277' (Eucalyptus urophylla $\times$ Eucalyptus camaldulensis), e nos híbridos 'Urocam' (Eucalyptus urophylla $\times$ E.camaldulensis) e 'Urograndis' (Eucalyptus urophylla $\times$ Eucalyptus grandis), no município de Cuiabá-MT, determinando-se a flutuação populacional da praga em espécie, clones e híbridos de eucaliptos, bem como de seus inimigos naturais.

\section{MATERIAL E MÉTODOS}

O trabalho foi realizado na Fazenda Jardim, localizada na Estrada MT $351 \mathrm{~km} \mathrm{42,} \mathrm{com} \mathrm{a}$ entrada da propriedade situada nas coordenadas $15^{\circ} 5^{\prime} 5.01^{\prime \prime} \mathrm{S}$ e $55^{\circ} 59^{\prime} 59.05^{\prime \prime} \mathrm{O}$, no município de Cuiabá, Estado de Mato Grosso.

O clima da região é do tipo AW (Koppen), abrangendo toda a Depressão Cuiabana, com temperatura média mensal de aproximadamente
25,7 ${ }^{\circ} \mathrm{C}$, precipitação pluvial anual média de $1.400 \mathrm{~mm}$, altitude de $800 \mathrm{~m}$ acima do nível do mar e solo do tipo areia quartzosa álica, de textura arenosa e destituído de minerais primários, pouco resistente ao intemperismo e baixa fertilidade (Conceição, 1997).

Para a realização dos estudos, foram utilizados: a espécie de Eucalyptus camaldulensis, os clones GG100 (E.urophylla $\times$ E. grandis) e MG1277 (E. urophylla $\times$ E. camaldulensis), e os híbridos Urocam (E. urophylla $\times$ E.camaldulensis $) \mathrm{e}$ Urograndis (E.urophylla $\times$ E. grandis), plantados no espaçamento de $3 \mathrm{~m} \times 3 \mathrm{~m}$ e procedentes de Alagoinhas-BA.

Os estudos foram realizados de junho de 2008 a julho de 2009. Para a espécie de E. camaldulensis, utilizou-se um talhão de 32,47 ha, com 26 meses de idade; para os híbridos 'Urocam' e 'Urograndis', utilizou-se um talhão de 30,86 ha e 14 meses de idade, e um talhão com 40,12 ha e idade de 19 meses, respectivamente; para os Clones GG100 e MG1277, utilizou-se um talhão com área de 39,60 ha e idade de 12 meses, e um talhão com de 42,42 ha com idade de 17 meses, respectivamente.

No monitoramento do adulto do psilídeo-deconcha, juntamente com seus inimigos naturais, foram utilizadas 50 armadilhas adesivas de cor amarela do tipo Biotrap ${ }^{\circledR}$, com as dimensões de $12 \mathrm{~cm} \times 11 \mathrm{~cm}$, descontando-se as bordas das armadilhas. Estas foram instaladas entre duas plantas a uma altura de 1,80 m da superfície do solo. Foram utilizadas dez armadilhas por ambiente de coleta e, a cada quinzena, as armadilhas foram substituídas. As armadilhas retiradas do campo foram envolvidas em papel filme e levadas para o Laboratório de Proteção Florestal, da Faculdade de Engenharia FlorestalLAPROFLOR, da Universidade Federal de Mato Grosso, para posterior contagem dos adultos e dos seus inimigos naturais.

A localização das armadilhas foram georreferenciadas com um GPS (Growth Position System) de navegação da marca Garmim ${ }^{\circledR}$, modelo Eritrex Legend.

Para o monitoramento dos ovos, ninfas e dos inimigos naturais do psilídeo-de-concha, foram realizadas coletas de folhas e ramos das plantas durante todo o período do estudo, juntamente com 
as coletas das armadilhas adesivas. As coletas de ramos foram realizadas a $2 \mathrm{~m}$ e $4 \mathrm{~m}$ de altura. Os ramos e as folhas coletados foram colocados em sacos plásticos, individualizados por altura e espécie de eucaliptos, e levados para o LAPROFLOR, onde foram acondicionados em freezer para posteriomente se realizar a quantificação de ovos, ninfas, adultos e de seus inimigos naturais.

Para as contagens, utilizou-se um microscópio estereoscópico, e as anotações das quantidades de insetos foram realizadas em uma ficha contendo as informações sobre o local, a data de coleta, a espécie estudada, as coordenadas das armadilhas e as informações sobre a coleta.

As armadilhas foram distribuídas no interior dos talhões seguindo a seguinte metodologia: nas laterais do talhão, foram instaladas três armadilhas a $30 \mathrm{~m}$ das bordaduras, equidistantes entre si. Nas extremidades do talhão, foi colocada uma armadilha no centro, distanciando-se $30 \mathrm{~m}$ das bordaduras, e no interior do talhão foram colocadas duas armadilhas próximas ao centro do talhão, paralelas às armadilhas colocadas nas laterais do talhão e equidistantes entre as duas extremidades, conforme a Figura 1.

Para o estudo da flutuação de G. brimblecombei e inimigos naturais, os dados coletados quinzenalmente foram transformados em mensais. A análise dos dados referentes ao estágio de desenvolvimento de G. brimblecombei (ovo, ninfa e adulto), aos inimigos naturais e às variáveis climáticas, foi realizada por meio da correlação de Pearson, com o auxílio do software estatístico SAEG 5.0.

\section{RESULTADOS E DISCUSSÃO}

\subsection{Flutuação populacional de adultos de Glycaspis brimblecombei em povoamentos de Eucalyptus camaldulensis nos Clones GG100 e MG1277 e nos Híbridos de Urocam e Urograndis}

Para a espécie E. camaldulensis, a quantidade de adultos de G.brimblecombei coletados foi maior no mês de agosto. A partir de setembro, as quantidades de indivíduos diminuíram gradualmente até não ser registrada sua presença de dezembro a junho, voltando a ocorrer em julho de 2009 (Figura 2).

No clone GG100, a praga ocorreu com poucos indivíduos de julho a outubro, e foi ausente nos demais meses; porém, no clone MG1277, a quantidade de adultos coletados foi maior no mês de agosto. A partir de setembro, a quantidade de

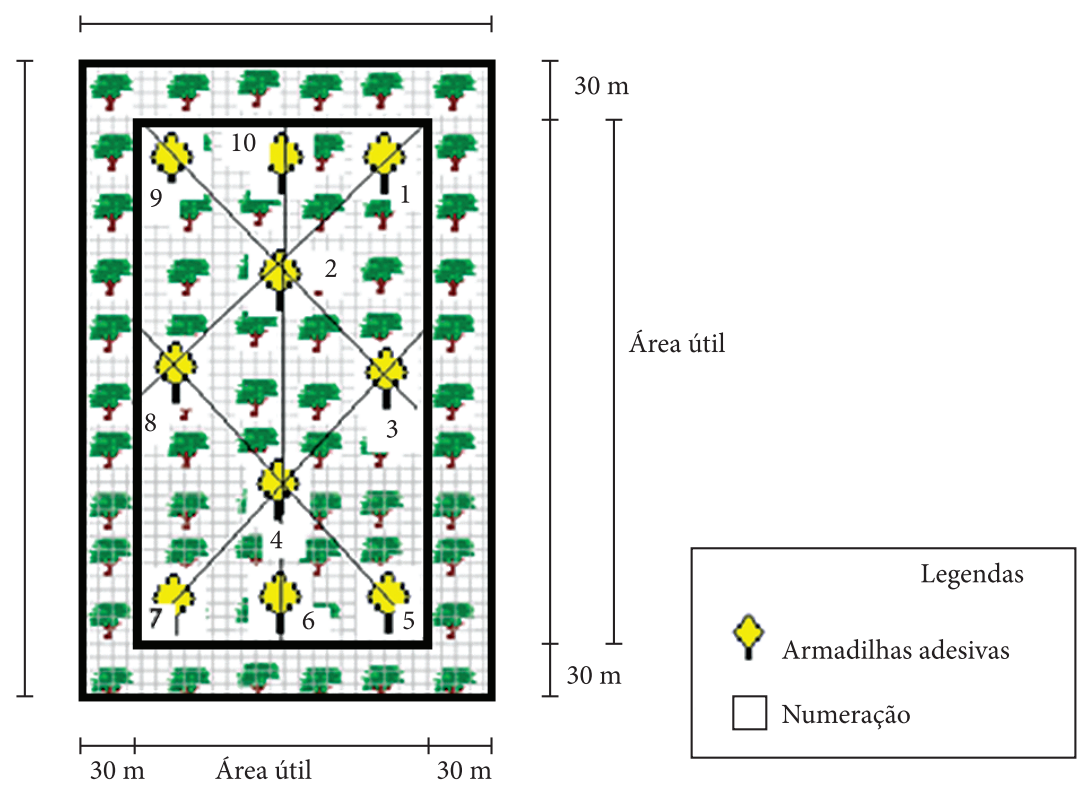

Figura 1. Disposição das armadilhas adesivas dentro dos talhões de Eucalyptus spp. na fazenda Jardim, Município de Cuiabá-MT.

Figure 1. Disposition of sticky traps inside the stands of Eucalyptus spp. Farm Jardim, Municipality of Cuiabá-MT. 


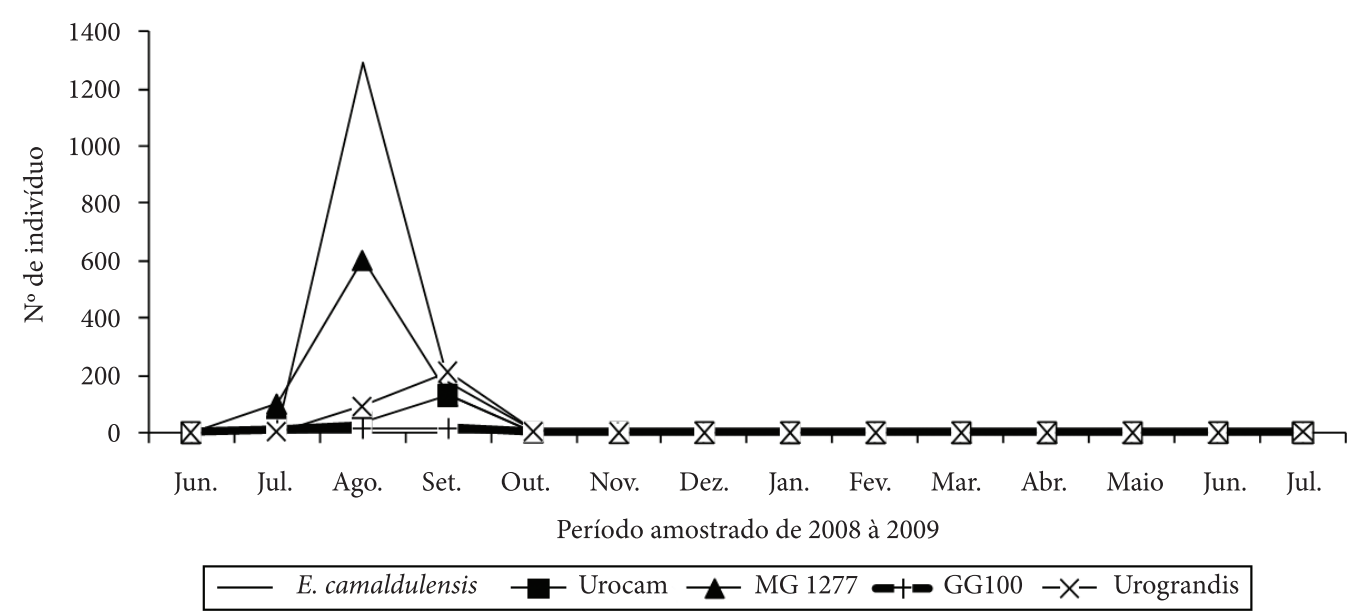

Figura 2. Flutuação populacional de adultos de Glycaspis brimblecombei em povoamentos de Eucalyptus camaldulensis nos Clones GG100 e MG1277, e nos Híbridos de Urocam e Urograndis. Cuiabá-MT, junho/2008 a julho/2009.

Figure 2. Population fluctuation of adults of Glycaspis brimblecombei in stands of Eucalyptus camaldulensis, in the clones GG100 and MG1277 and hybrids Urocam and urograndis. Cuiabá-MT, July 2008 to June 2009.

indivíduos diminuiu gradualmente até não ser registrada sua presença de novembro a junho de 2009 (Figura 2).

No híbrido Urocam, a quantidade de adultos de G. brimblecombei coletados foi maior no mês de setembro. Nos meses de outubro a dezembro, foi registrado apenas um indivíduo em cada mês, sendo ausente para os demais meses. No híbrido Urograndis, a quantidade de adultos coletados foi maior no mês de setembro. Nos meses de outubro a junho, não foi registrada a presença de indivíduos adultos, ocorrendo novamente em julho (Figura 2).

\subsection{Quantidade de ovos coletados de Glycaspis brimblecombei em povoamentos de Eucalyptus camaldulensis, dos Clones GG100 e MG1277, e dos Híbridos Urocam e Urograndis.}

Para a espécie E. camaldulensis, a quantidade de ovos coletados de G. brimblecombei foi maior em agosto. A partir de setembro, a quantidade de ovos começou a diminuir até não ser registrada sua presença de dezembro a junho, voltando a ocorrer em julho de 2009 (Figura 3).

No clone GG100, a quantidade de ovos de G. brimblecombei coletados foi maior em agosto. A partir de setembro, a quantidade de ovos começou a diminuir até não ser registrada sua presença de dezembro a junho, voltando ocorrer em julho de 2009. No clone MG1277, a quantidade de ovos coletados foi maior em agosto. A partir de setembro, a quantidade de ovos começou a diminuir gradualmente até não ser registrada sua presença de dezembro a junho, voltando a ocorrer em julho de 2009 (Figura 3).

A quantidade de ovos de G. brimblemcobei coletados em folhas do híbrido Urocam foi maior em agosto. A partir de setembro, a quantidade de ovos começou a diminuir gradualmente até não serem registradas as posturas de G. brimblecombei no período de dezembro a junho, voltando a ocorrer em julho de 2009. No híbrido Urograndis, a quantidade de ovos coletados foi maior em agosto. A partir de setembro, a quantidade de ovos de G. brimblecombei começou a diminuir gradualmente até não serem registradas posturas no período de dezembro a junho, voltando a ocorrer em julho de 2009 (Figura 3).

\subsection{Ninfas de G. brimblecombei coletadas em folhas de Eucalyptus camaldulensis, dos Clones GG100 e MG1277 e dos Híbridos Urocam e Urograndis}

Para a espécie E. camaldulensis, a quantidade de ninfas de $G$. brimblecombei coletadas de G.brimblecombei foi maior em agosto e, a partir de setembro, começou a diminuir até não ser registrada 
sua presença de dezembro a junho, voltando a ocorrer em julho de 2009 (Figura 4).

Ninfas de G. brimblecombei foram coletadas em maior quantidade no talhão de clone GG100 durante o mês de agosto. A partir de setembro, a quantidade de ninfas começou a diminuir até não ser registrada sua presença de dezembro a junho, voltando a ocorrer em julho de 2009. A quantidade de ninfas coletadas em folhas do clone MG1277 foi maior em agosto e, a partir de setembro, a quantidade começou a diminuir gradualmente até não ser registrada sua presença de dezembro a julho, voltando a ocorrer em julho de 2009 (Figura 4).

No híbrido Urocam, a quantidade de ninfas de G. brimblecombei coletadas foi maior em agosto. A partir de setembro, a quantidade de ninfas começou a diminuir gradualmente até não ser registrada sua presença de dezembro a junho, voltando a ocorrer em julho de 2009. No híbrido Urograndis, a quantidade de ninfas coletadas foi maior em setembro. A partir de outubro, a quantidade de ninfas começou a diminuir gradualmente até não ser registrada sua

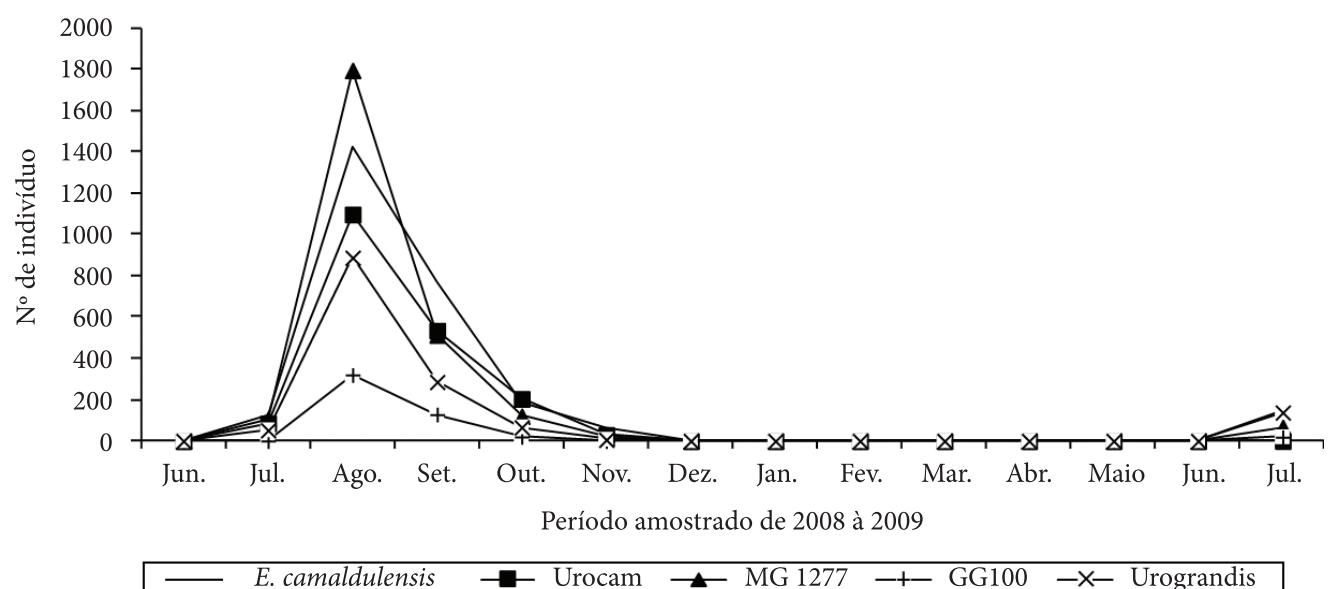

Figura 3. Quantidade de ovos coletados de Glycaspis brimblecombei em povoamentos de Eucalyptus camaldulensis, dos Clones GG100 e MG1277, e dos Híbridos Urocam e Urograndis. Cuiabá-MT, junho/2008 a julho/2009.

Figure 3. Quantity of eggs collected of Glycaspis brimblecombei in stands of Eucalyptus camaldulensis, in clones GG100 and MG1277 and hybrids Urocam and urograndis. Cuiabá-MT, July 2008 to June 2009.

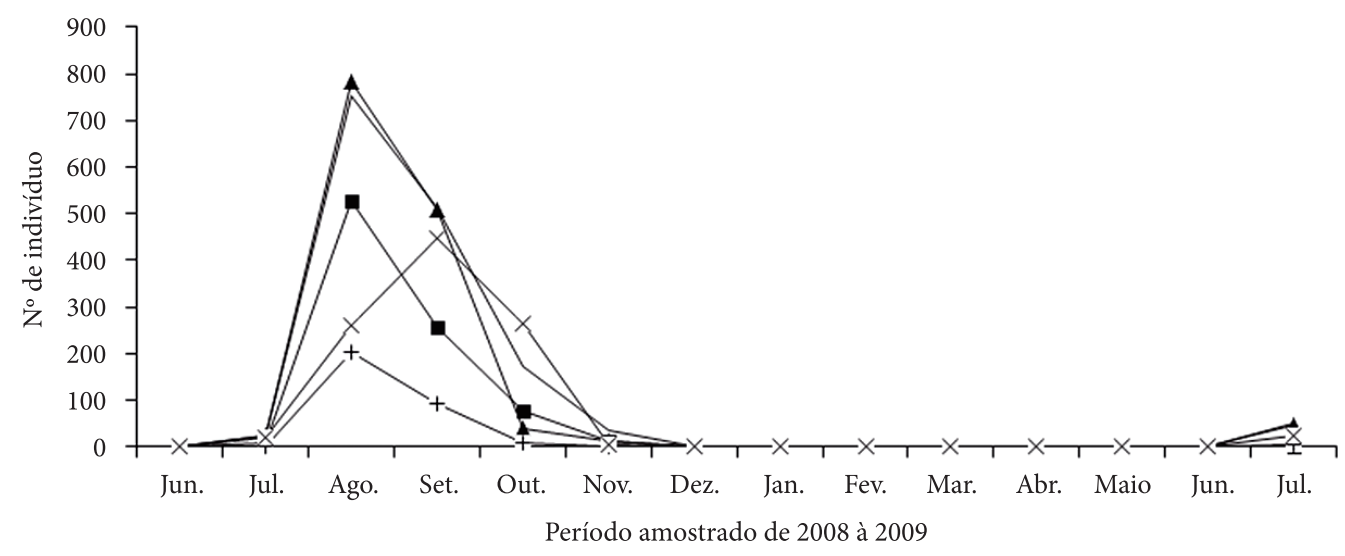

- E. camaldulensis $\rightarrow$ Urocam $\rightarrow$ MG $1277 \rightarrow$ GG100 $\rightarrow \times-$ Urograndis

Figura 4. Quantidade de ninfas coletadas de Glycaspis brimblecombei em folhas de Eucalyptus camaldulensis, dos Clones GG100 e MG1277, e dos Híbridos Urocam e Urograndis. Cuiabá-MT, junho/2008 a julho/2009.

Figure 4. Quantity of nymphs collecteds of Glycaspis brimblecombei on leaves of Eucalyptus camaldulensis, clones GG100 and MG1277, hybrids Urocam and Urograndis. Cuiabá-MT, July 2008 to June 2009. 
presença de dezembro a junho, voltando a ocorrer em julho de 2009 (Figura 4).

O levantamento populacional do psilídeo-deconcha foi superior no período de seca, em todos os estágios de ovo, ninfa e adulto, principalmente nos meses de agosto e setembro, em todos os povoamentos de eucaliptos estudados.

Resultados semelhantes foram obtidos por Paine et al. (2000), Ramirez et al. (2003), Wilcken et al. (2003), Ferrreira Filho (2005), Ferreira Filho et al. (2008) e Ferreira et al. (2009), com elevadas temperaturas no período de seca, propiciando aumento na quantidade de indivíduos de G. brimblecombei, e diminuindo tal quantidade com a chegada das chuvas e a diminuição da temperatura.

\subsection{Flutuação populacional de}

Psyllaephagus bliteus e de outros inimigos naturais de Glycaspis brimblecombei

A flutuação populacional do parasitóide P. bliteus apresentou-se de forma irregular, todavia demonstrando uma tendência de aumento da sua população nos meses de seca, época de maior ocorrência do seu hospedeiro. Adultos dos parasitoides coletados em maior quantidade ocorreram durante o mês de junho de 2008. No ano de 2009, houve uma queda na população de $P$. bliteus em relação ao ano anterior (Figura 5).
Predadores de G. brimblecombei coletados apresentaram maiores quantidades de indivíduos em setembro. De outubro até dezembro, a quantidade de adultos decresceu, aumentando em janeiro e registrando, em fevereiro, um aumento dos predadores, chegando próximo ao registro do mês de setembro. Este fato pode estar correlacionado com o aumento de presas, já que os predadores não são específicos ao psilídeo-de-concha e também com o fato de que, em março de 2009, foi registrada desfolha por lagartas das espécies Thyrinteina arnobia, Nystalea nyseus, Blera sp. e Sarsina violascens, na área amostrada. Nos demais meses, a quantidade de indivíduos diminuiu gradualmente (Figura 6).

Durante os 14 meses de estudo, foram coletados em folhas, ramos e armadilhas adesivas predadores da família Coccinelidae, adultos de Chrysoperla sp. (Neuroptera). Foram constatadas algumas aranhas próximas à ocorrência do psilídeo-de-concha e também nas armadilhas adesivas.

Resultados similares também foram constatados por Dezane et al. (2006), verificando uma sincronia na flutuação populacional da praga e do parasitóide P. bliteus. Quanto aos predadores, foi observada maior ocorrência de coccinelídeos do que crisopídeos, e verificou-se que os predadores tiveram picos em outubro e dezembro de 2005, quando a população da praga apresentava menor quantidade.

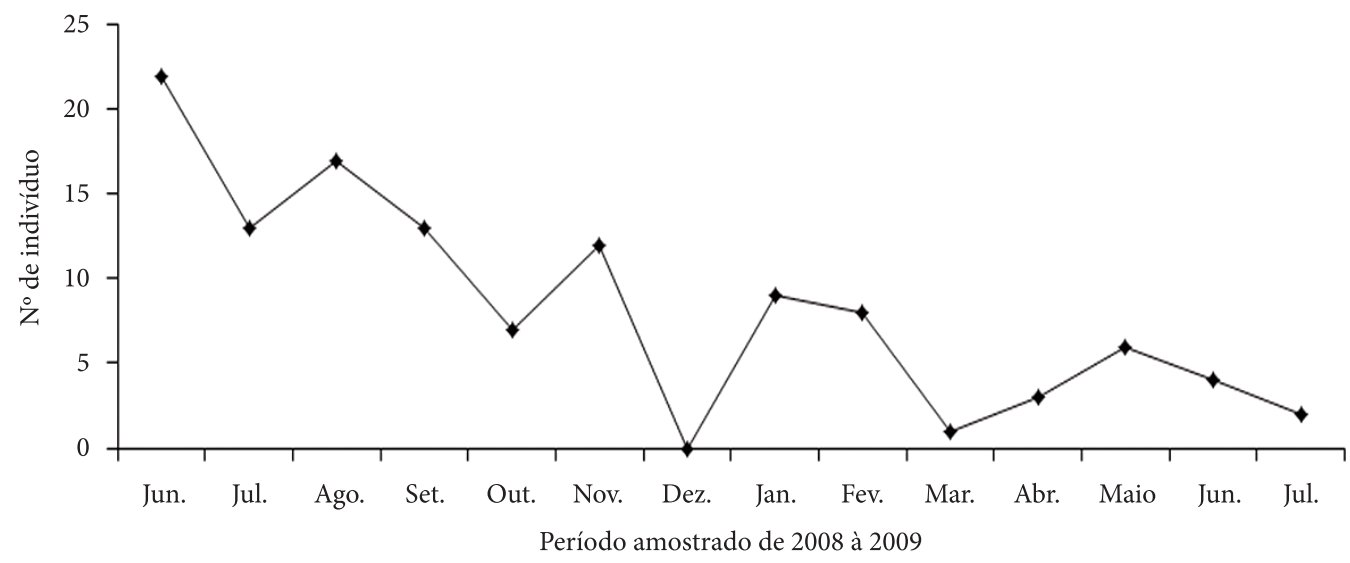

Figura 5. Flutuação populacional do parasitóide Psyllaephagus bliteus (Hymenoptera: Encyrtidae). Cuiabá-MT, Junho/2008 a Julho/2009.

Figure 5. Populacional Fluctuation of Psyllaephagus bliteus parasitoid (Hymenoptera: Encyrtidae). Cuiabá-MT, July 2008 to June 2009. 


\subsection{Influência das variáveis meteorológicas na população de Glycaspis brimblecombei e dos inimigos naturais}

A flutuação populacional dos indivíduos de G.brimblecombei apresentou correlação positiva e diretamente proporcional com a temperatura máxima, e correlação negativa e inversamente proporcional com a umidade relativa do ar; chegouse a essas correlções por meio da análise de correlação de Pearson para todos os estágios do psilídeode-concha na espécie e nos clones e híbridos dos eucaliptos avaliados (Tabela 1).

A temperatura mínima foi significativa apenas para a quantidade de indivíduos adultos de $G$. brimblecombei coletados no clone GG100, sendo tal quantidade inversamente proporcional a esta variável. A temperatura média não interferiu significativamente na população de G. brimblecombei, nas fases de desenvolvimento e nos plantios de eucalipto avaliados. (Tabela 1).

Do mês de setembro até o mês de dezembro de 2008, houve uma pequena variação nas temperaturas; no entanto, a quantidade de indivíduos coletados começou a diminuir até ocorrer ausência de indivíduos de janeiro a maio de 2009. Nos meses de junho e julho, os indivíduos voltaram a ser registrados, sendo as temperaturas nesses meses similares às do ano anterior (Figura 7).

Pela análise de correlação de Pearson dos parâmetros temperaturas máximas, médias e mínimas, precipitação e umidade relativa do ar, não se apresentaram diferenças significativas para P. bliteus e predadores (Tabela 2).

Com o aumento das temperaturas máxima, média e mínima de julho até agosto de 2008, registraram-se as maiores quantidades de P. bliteus coletados. Nos demais meses, setembro de 2008 a julho de 2009, as alterações destas variáveis não influenciaram no aumento da quantidade de indivíduos coletados (Figura 8).

Os predadores foram coletados em maiores quantidades em setembro de 2008, com as temperaturas máxima, média e mínima em $35,7^{\circ} \mathrm{C}$; $16,8^{\circ} \mathrm{C}$, e $26,3^{\circ} \mathrm{C}$, respectivamente (Figura 8).

Favaro (2006) encontrou diferenças significativas e diretamente proporcionais ao correlacionar as temperaturas máxima, mínima e média em relação aos dados dos adultos do psilídeo, e não significativas para as quantidades de ovos e ninfas. Também encontrou diferenças significativas e diretamente proporcionais para a quantidade do parasitóide P.bliteus e não significativa para a quantidade dos outros inimigos naturais de G. brimblecombei, coletados no talhão de Eucalyptus sp.

Mateus et al. (2007) constataram sincronia entre G. brimblecombei e P. bliteus, indicando que o inimigo natural é eficaz para ser utilizado no controle do psilídeo-de-concha em plantios de Eucaliptos; no entanto, em relação aos dados de predadores (coccinelídeos e vespídeos), a correlação mantevese baixa, indicando ausência de sincronismo entre a população da praga e dos seus possíveis predadores.

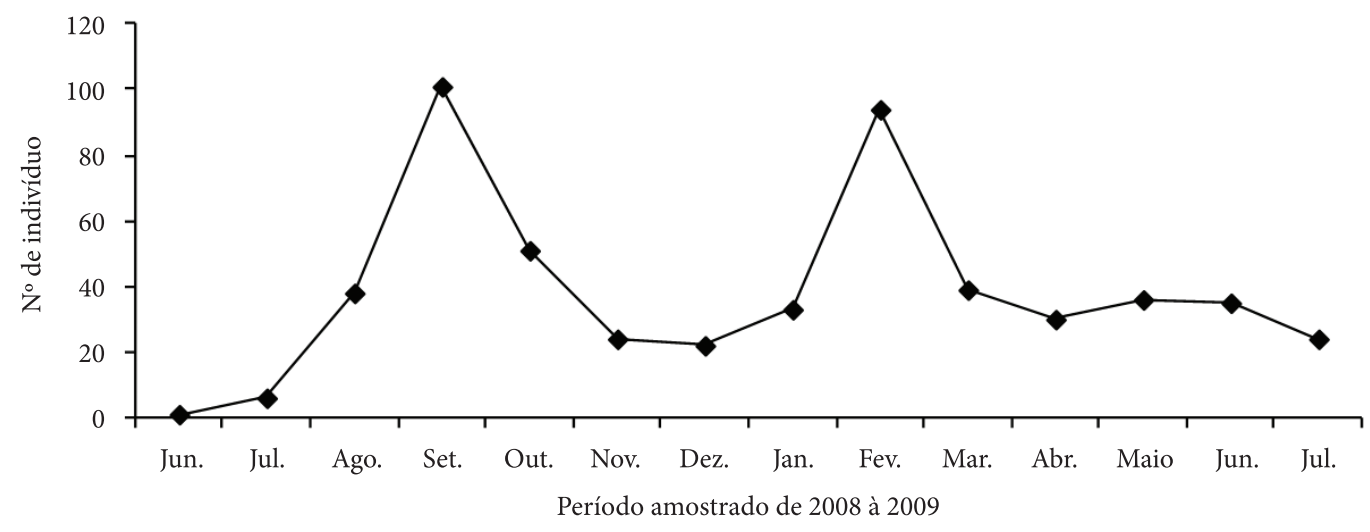

Figura 6. Flutuação populacional dos predadores de Glycaspis brimblecombei. Cuiabá-MT, Junho/2008 a Julho/2009. Figure 6. Populational Flutuation of the Glycaspis brimblecombei Predators. Cuiabá-MT, July 2008 to June 2009. 
Tabela 1. Correlação de Pearson para os diferentes estágios de Glycaspis brimblecombei em Eucalyptus camaldulensis, nos clones GG100 e MG1277, e nos híbridos Urocam e Urograndis. Cuiabá-MT, junho/2008 a julho/2009.

Table 1. Pearson correlation to different stages of Glycaspis brimblecombei in Eucalyptus camaldulensis, on clones GG100 and MG1277, the hybrids Urocam and Urograndis. Cuiabá-MT, July 2008 to June 2009.

\begin{tabular}{|c|c|c|c|c|c|c|c|}
\hline Hospedeiro & \multicolumn{2}{|c|}{ Estágio } & $\begin{array}{l}\text { Temperatura } \\
\text { máxima }\end{array}$ & $\begin{array}{l}\text { Temperatura } \\
\text { mínima }\end{array}$ & $\begin{array}{c}\text { Temperatura } \\
\text { média }\end{array}$ & UR\% & $\mathbf{P P}(\mathbf{m m})$ \\
\hline \multirow{6}{*}{ E. camaldulensis } & \multirow{2}{*}{ Ovo } & $(\mathrm{r})$ & $0,6925^{\star}$ & $-0,3832$ & 0,1042 & $-0,7863^{*}$ & $-0,3591$ \\
\hline & & $(\mathrm{P})$ & 0,003 & 0,881 & 0,3615 & 0,0007 & 0,1037 \\
\hline & \multirow{2}{*}{ Ninfa } & $(\mathrm{r})$ & $0,6918^{*}$ & $-0,3394$ & 0,1425 & $-0,7544^{*}$ & $-0,3234$ \\
\hline & & $(\mathrm{P})$ & 0,0031 & 0,1175 & 0,3135 & 0,0009 & 0,1297 \\
\hline & \multirow{2}{*}{ Adulto } & $(\mathrm{r})$ & $0,5463^{\star}$ & $-0,2693$ & 0,1115 & $-0,6928^{*}$ & $-0,3248$ \\
\hline & & (P) & 0,0216 & 0,176 & 0,3522 & 0,030 & $-0,1286$ \\
\hline \multirow{6}{*}{ GG100 } & \multirow{2}{*}{ Ovo } & $(\mathrm{r})$ & $0,6349^{*}$ & $-0,3259$ & 0,1181 & $-0,7546^{\star}$ & $-0,3411$ \\
\hline & & $(\mathrm{P})$ & 0,0074 & 0,1277 & 0,3438 & 0,0009 & 0,1163 \\
\hline & \multirow{2}{*}{ Ninfa } & $(\mathrm{r})$ & $0,6349^{*}$ & $-0,3173$ & 0,1243 & $-0,7539^{*}$ & $-0,3262$ \\
\hline & & (P) & 0,0074 & 0,1345 & 0,336 & 0,0009 & 0,1275 \\
\hline & \multirow{2}{*}{ Adulto } & $(\mathrm{r})$ & $0,6327^{\star}$ & $-0,5595^{\star}$ & 0,465 & $-0,6747^{\star}$ & $-0,4084$ \\
\hline & & (P) & 0,0076 & 0,0187 & 0,187 & 0,0041 & 0,0735 \\
\hline \multirow{6}{*}{ MG1277 } & \multirow{2}{*}{ Ovo } & (r) & $0,7013^{\star}$ & $-0,376$ & 0,071 & $-0,7411^{*}$ & $-0,3557$ \\
\hline & & $(\mathrm{P})$ & 0,0026 & 0,1264 & 0,3578 & 0,012 & 0,106 \\
\hline & \multirow{2}{*}{ Ninfa } & $(\mathrm{r})$ & $0,6202^{*}$ & $-0,3603$ & 0,1091 & $-0,7627^{\star}$ & $-0,3273$ \\
\hline & & (P) & 0,003 & 0,1029 & 0,3552 & 0,0008 & 0,1267 \\
\hline & \multirow{2}{*}{ Adulto } & $(\mathrm{r})$ & $0,6685^{\star}$ & $-0,3557$ & 0,0673 & $-0,7624^{\star}$ & $-0,3772$ \\
\hline & & (P) & 0,0045 & 0,106 & 0,4096 & 0,0016 & 0,0919 \\
\hline \multirow{6}{*}{ Urocam } & \multirow{2}{*}{ Ovo } & $(\mathrm{r})$ & $0,5968^{\star}$ & $-0,3243$ & 0,1401 & $-0,7563^{*}$ & $-0,3393$ \\
\hline & & (P) & 0,0121 & 0,129 & 0,3164 & 0,0009 & 0,1176 \\
\hline & \multirow{2}{*}{ Ninfa } & $(\mathrm{r})$ & $0,6670^{*}$ & $-0,3047$ & 0,1478 & $-0,7507^{\star}$ & $-0,3195$ \\
\hline & & $(\mathrm{P})$ & 0,0046 & 0,1448 & 0,3071 & 0,0001 & 0,1327 \\
\hline & \multirow{2}{*}{ Adulto } & $(\mathrm{r})$ & $0,6519^{*}$ & $-0,3605$ & 0,0256 & $-0,5239$ & $-0,1941$ \\
\hline & & (P) & 0,0058 & 0,1027 & 0,4654 & 0,0272 & 0,2531 \\
\hline \multirow{6}{*}{ Urograndis } & \multirow{2}{*}{ Ovo } & $(\mathrm{r})$ & $0,5385^{\star}$ & $-0,3848$ & 0,076 & $-0,7576^{*}$ & $-0,3913$ \\
\hline & & (P) & 0,0235 & 0,0872 & 0,3981 & 0,0008 & 0,0832 \\
\hline & \multirow{2}{*}{ Ninfa } & $(\mathrm{r})$ & $0,6506^{*}$ & $-0,2582$ & 0,1714 & $-0,5964^{*}$ & $-0,2413$ \\
\hline & & (P) & $0,005^{\star}$ & 0,1864 & 0,279 & 0,0122 & 0,203 \\
\hline & \multirow{2}{*}{ Adulto } & $(\mathrm{r})$ & 0,5611 & $-0,3055$ & 0,0889 & $-0,5830^{*}$ & $-0,1797$ \\
\hline & & $(\mathrm{P})$ & 0,0184 & 0,1441 & 0,3812 & 0,0143 & 0,2694 \\
\hline
\end{tabular}

*Significativo ao nível de $5 \%$ de Probabilidade; (r) correlação de Pearson; (P) probabilidade.

Ferreira Filho et al. (2008) verificaram que as populações de G. brimblecombei e P. bliteus possuem em plantio de Eucalyptus spp. correlação inversamente proporcional em relação à temperatura. Todavia, entre tais populações, não houve correlação com a precipitação no período avaliado.

Em junho de 2008, foi registrada a umidade relativa do ar de $94,7 \%$, sendo o maior valor desta variável. Nos meses de julho, agosto e setembro de 2008, foram registradas umidades relativas do ar de $87,8 \% ; 81,5 \%$, e $80,9 \%$, respectivamente, e em junho e julho de 2009 foram registradas $87,4 \%$ e
$87,8 \%$, respectivamente, constituindo os menores valores durante todo o experimento (Figura 9). Para estes dados, foram constatados que quanto maior a umidade relativa, menores foram as quantidades de indivíduos coletados.

A precipitação total foi nula nos meses de junho a agosto de 2008, período em que foi constatada maior quantidade dos indivíduos de G. brimblecombei coletados e também de P. bliteus. Nos demais meses em que ocorreram precipitações variando entre 6 e $250 \mathrm{~mm}$, as populações do psilídeo e de $P$. bliteus 
Tabela 2. Correlação de Pearson para Psyllaephagus bliteus e predadores de Glycaspis brimblecombei. Cuiabá-MT, junho/2008 a julho/2009.

Table 2. Pearson correlation to Psyllaephagus bliteus and predators of Glycaspis brimblecombei. Cuiabá-MT, July 2008 to June 2009 .

\begin{tabular}{llccccc}
\multicolumn{1}{c}{ População } & & $\begin{array}{c}\text { Temperatura } \\
\text { máxima }\end{array}$ & $\begin{array}{c}\text { Temperatura } \\
\text { mínima }\end{array}$ & $\begin{array}{c}\text { Temperatura } \\
\text { média }\end{array}$ & UR\% & PP(mm) \\
\multirow{2}{*}{ P. bliteus } & $(\mathrm{r})$ & $-0,0135$ & $-0,4169$ & $-0,3782$ & $-0,039$ & $-0,4373$ \\
\multirow{2}{*}{ Predadore } & $(\mathrm{P})$ & $0,4817^{\mathrm{ns}}$ & $0,069^{\mathrm{ns}}$ & $0,0912^{\mathrm{ns}}$ & $0,4474^{\mathrm{ns}}$ & $0,059^{\mathrm{ns}}$ \\
& $(\mathrm{r})$ & 0,2915 & 0,2971 & 0,4501 & $-0,426$ & 0,3172 \\
\hline & $(\mathrm{P})$ & $0,156^{\mathrm{ns}}$ & $0,1512^{\mathrm{ns}}$ & $0,0532^{\mathrm{ns}}$ & $0,0644^{\mathrm{ns}}$ & $0,1346^{\mathrm{ns}}$ \\
\hline
\end{tabular}

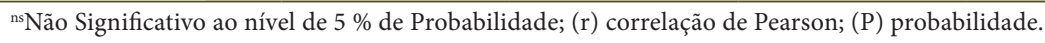

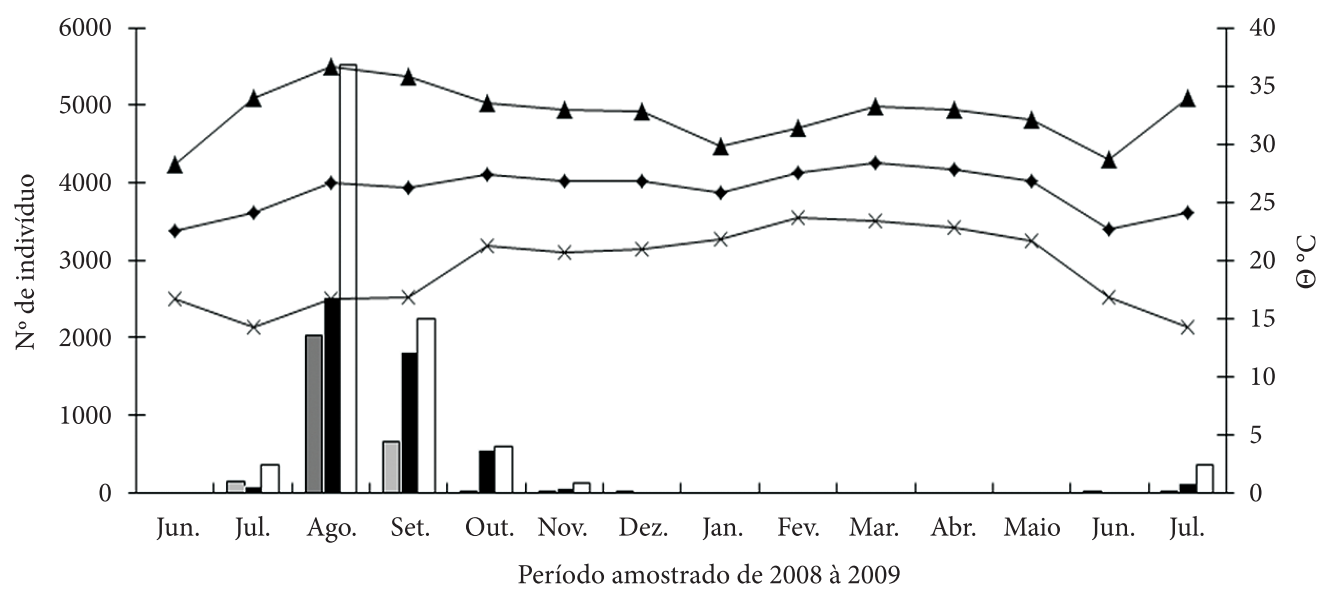

\begin{tabular}{|c|c|c|c|c|c|}
\hline$\square$ Adulto & Ninfa & $\square$ Ovo & Ł-Tmáx & * Tmín & $\rightarrow$ Tméd \\
\hline
\end{tabular}

Figura 7. Temperaturas máximas, médias e mínimas, e flutuação populacional de todos os estágios de Glycaspis brimblecombei. Cuiabá-MT, Junho/2008 a Julho/2009.

Figure 7. Maximum, Average, Minimum Temperature and Populational Fluctuation of all stages of Glycaspis brimblecombei. Cuiabá-MT July 2008 to June 2009.

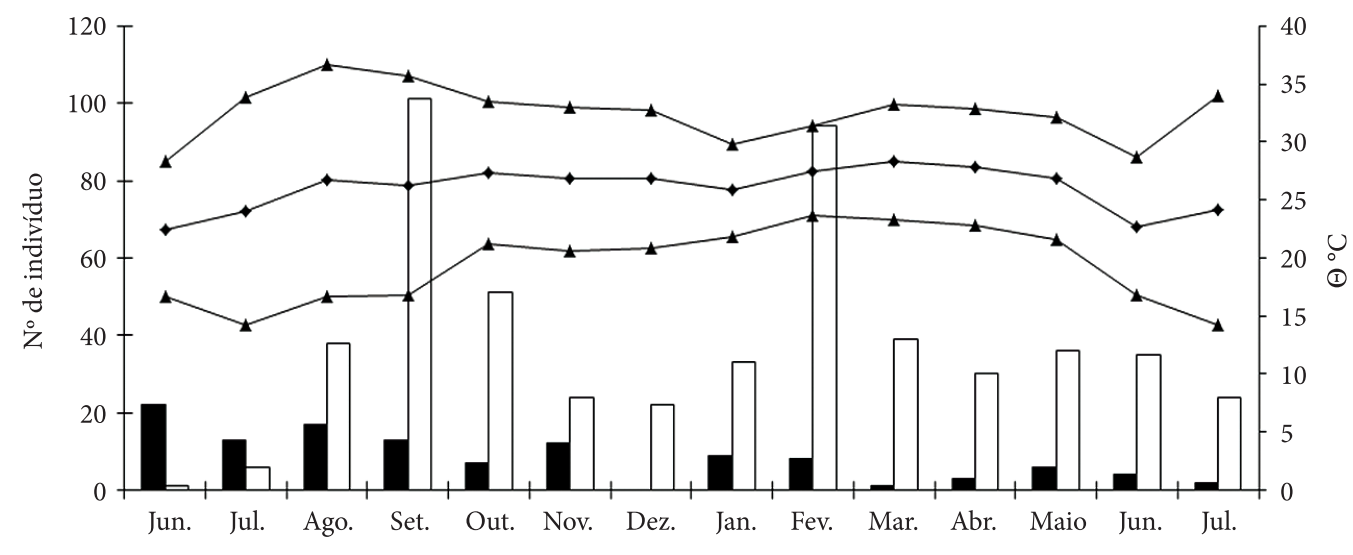

Período amostrado de 2008 à 2009

- Psyllaephagus bliteus $\square$ Inimigos naturais $\quad$ Tmáx $\quad$ Tmín $\rightarrow$ Tméd

Figura 8. Temperaturas máxima, média e mínima, e flutuação populacional de Psyllaephagus bliteus e predadores de Glycaspis brimblecombei. Cuiabá-MT, Junho/2008 a Julho/2009.

Figure 8. Temperature Maximum, Average, Minimum and Floating Population of Psyllaephagus bliteus and Glycaspis brimblecombei Predators. Cuiabá, July 2008 to June 2009. 


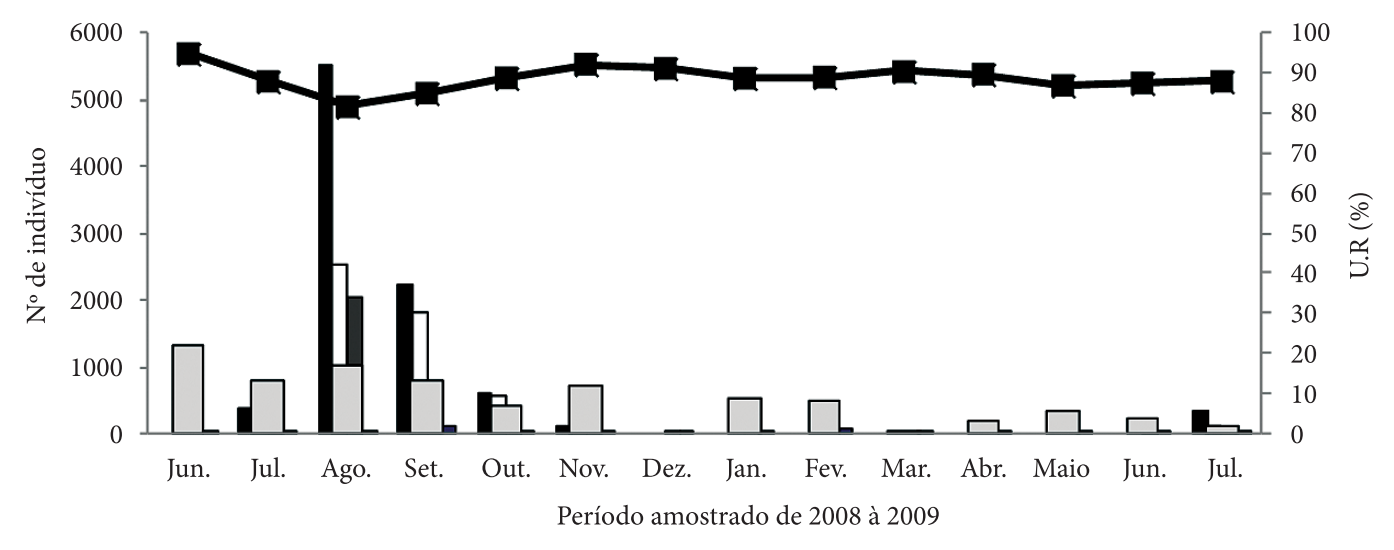

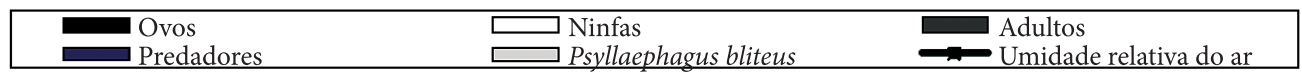

Figura 9. Influência da umidade relativa do ar nos diferentes estágios de G.brimblecombei e inimigos naturais, Cuiabá-MT, Junho/2008 a Julho/2009.

Figure 9. Influence of Relative Humidity at different stages of G.brimblecombei and natural enemies, Cuiabá-MT, July 2008 to June 2009.

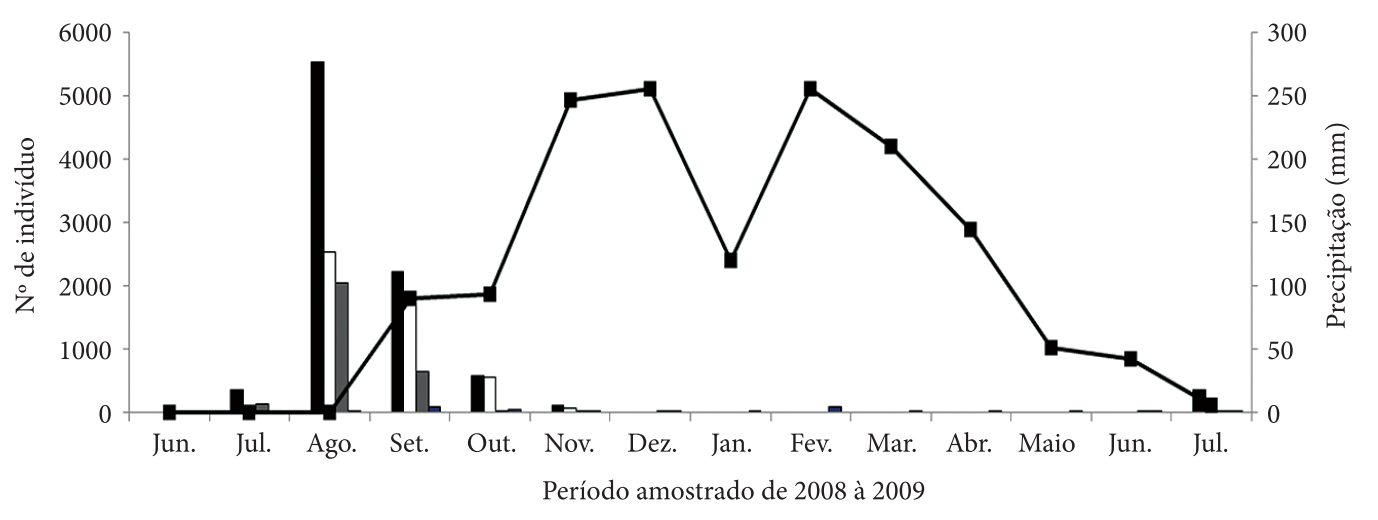

Psyllaephagus bliteus $\square$ Ovos $\square$ Ninfas $\square$ Adultos $\square$ Predadores $\quad$ - Precipitação total

Figura 10. Influência da precipitação total nos diferentes estágios de G.brimblecombei e inimigos naturais, CuiabáMT, Junho/2008 a Julho/2009.

Figure 10. Influence of Total Precipitation at different stages of G.brimblecombei and natural enemies, Cuiabá-MT, July 2008 to June 2009.

diminuíram, reaparecendo em junho e julho de 2009, com o decréscimo da precipitação (Figuras 10).

A população dos predadores de G. brimblecombei aumentou no início do período de chuva, em setembro de 2008 e em fevereiro de 2009 (Figura 10).

Sookar et al. (2003) afirmaram que existe uma relação entre a redução das populações de $G$. brimblecombei em plantio de Eucalyptus spp. com a chegada de períodos de chuvas intensas e que estudos realizados na Austrália com Glycaspis baileyi indicaram que o clima é um parâmetro a ser considerado na regulação da população destes insetos e de outras espécies de psilídeos.

O estresse hídrico observado em plantas de E. camaldulensis induz um aumento na densidade média de ovos e de ninfas de G. brimblecombei. Este fato pode sugerir um padrão de seleção do sugador quanto à qualidade alimentar da planta hospedeira. 


\section{CONCLUSÕES}

O período da seca e a temperatura máxima são fatores favoráveis ao aumento da população de Glycapis brimblecombei, sendo que o inverso ocorre para a umidade relativa do ar.

E. camaldulensis e o clone MG1277 (E. urophylla $\times$ E. camaldulensis) são hospedeiros favoráveis ao aumento da população de G. brimblecombei; diversamente, o Clone GG100 é o hospedeiro menos favorável ao aumento da população de G. brimblecombei.

\section{STATUS DA SUBMISSÃO}

Recebido: 25/10/2011

Aceito: 30/11/2012

Publicado: 28/02/2013

\section{AUTOR(ES) PARA CORRESPONDÊNCIA}

\section{Alex Lima da Silva}

Faculdade de Engenharia Florestal - FENF, Universidade Federal de Mato Grosso - UFMT, Av. Fernando Correa da Costa, s/n, Coxipó, CEP 78060-900, Cuiabá, MT, Brasil e-mail: alexperplex@hotmail.com

\section{Otávio Peres Filho}

Faculdade de Engenharia Florestal - FENF, Universidade Federal de Mato Grosso - UFMT, Av. Fernando Correa da Costa, s/n, Coxipó, CEP 78060-900, Cuiabá, MT, Brasil e-mail: peres@ufmt.br

\section{REFERÊNCIAS}

Conceição PN. Manejo de Bacias Hidrográficas do Rio Coxipó-Açú para conservação dos Recursos Hídricos. Brasília: Abeas/MMa/SRH/UFMT; 1997. 127 p.

Dezane AFS, Sá LA, Almeida GR. Estudo da Flutuação de populacional do psilídeo-de-concha Glycaspis brimblecombei (Hemiptera: Psyllidae) e de seus inimigos naturais em florestas de eucalipto na região de Mogi Guaçu, SP. In: Jornada Acadêmica da Embrapa meio Ambiente, 2006, Jaguariúna. Jaguariúna: Embrapa Meio Ambiente, 2006. p. 57-60.

Favaro RM. Aspectos bionômicos de Glycaspis brimblecombei (Moore, 1964) (Hemiptera: Psyllidae) $e$ seu controle com fungos entomopatogênicos [dissertação]. Curitiba: Universidade Federal do Paraná; 2006.

Ferreira Filho PJ. Estudo de populações do psilídeo-deconcha Glycaspis brimblecombei (Hemiptera: Psyllidae) e de seu parasitóide Psyllaephagus bliteus (Hymenoptera: Encyrtidae) em floresta de Eucalyptus camaldulensis por dois métodos de amostragem [dissertação]. Botucatu: Universidade Estadual Paulista Julio Mesquita Filho; 2005.

Ferreira Filho PJ, Wilcken CF, Oliveira NC, Dal Pogetto MHFA, Lima ACV. Dinâmica populacional do psilídeo-de-concha Glycaspis brimblecombei (Moore, 1964) (Hemiptera: Psyllidae) e de seu parasitóide Psyllaephagus bliteus (Hymenoptera: Encyrtidae) em floresta de Eucalyptus camaldulensis. Ciência Rural 2008, 38(8): 2109-2114. http://dx.doi. org/10.1590/S0103-84782008000800003

Ferreira RA, Blaziza AAB, Anzolin MG, FirminoWinckler DC. Flutuação populacional do psilídeo-deconcha, Glycaspis brimblecombei Moore (Hemiptera: Psyllidae) em Eucalyptus spp. No município de Graça, SP. [cited 2009 Sept 12] Avaliable from: http://www.revista. inf.br.

Firmino DC. Biologia do psilídeo-de-concha Glycaspis brimblecombei Moore (Hemiptera: Psyllidae) em diferentes espécies de eucalipto e em diferentes temperaturas [dissertação]. Botucatu: Universidade Estadual Paulista Julio Mesquita Filho; 2004.

Mateus GS, Wilcken CF, Abdalla MC, Dal Pogetto MHFA, Couto EB. Monitoramento do psilídeode-concha Glycaspis brimblecombei (Hemiptera: Psyllidae) e de seus inimigos naturais em florestas clonais de eucalipto na região de Botucatu-SP. In: Simpósio Internacional de Iniciação Científica; 2007; Pirassununga. Pirassununga, 2007.

Paine TD, Dahlsten DL, MillarJG, Hoddle MS, Hanks LM. UC scientists apply IPM techniques to new eucalyptus pests. California Agriculture 2000; 54(6): 8-13. http:// dx.doi.org/10.3733/ca.v054n06p8

Ramirez ALG. Fluctuacion poblacional del psilido del eucalipto Glycaspis brimblecombei y el efecto del control biológico con la avispa parasitóide Psyllaephagus bliteus [dissertação]. Cuatitlan Izcalli: Ingeniera AgrícolaFacultad de Estudos Superiores Cuautitlan; 2003.

Sookar P, Seewooruthun SI, Ramkhelawon D. The redgum lerp psyllidae, Glycaspis brimblecombei, a new pest of Eucalyptus sp. in Mauritius. AMAS 2003; 327-332.

Wilcken CF, Couto EB, Orlato C, Ferreira Filho PJ, Firmino DC. Ocorrência do psilídeo-de-concha (Glycaspis brimblecombei) em florestas de eucalipto no Brasil. Circular Técnica Ipef, 2003; 201, 1-11 [cited 2007 Oct 8] Avaliable from: http://www.ipef.br/publicacoes/ ctecnica. 